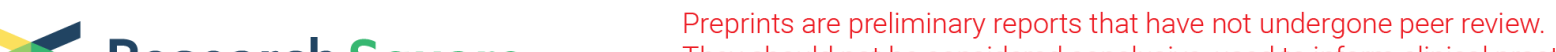 Research Square They should not be considered conclusive, used to inform clinical practice, or referenced by the media as validated information.
}

\section{Evaluation of Carotid Artery Elastic Function Using Ultrafast Pulse Wave Velocity and Related Influential Factors in Patients with Rheumatoid Arthritis}

\section{Yihan Li}

First Affiliated Hospital of Jinzhou Medical University Jian Zhang

First Affiliated Hospital of Jinzhou Medical University

\section{Xin An}

First Affiliated Hospital of Jinzhou Medical University

Jihui Li

First Affiliated Hospital of Jinzhou Medical University

Shanshan Shi

First Affiliated Hospital of Jinzhou Medical University

\section{Siyang Ma}

First Affiliated Hospital of Jinzhou Medical University

\section{Cungang Wu}

First Affiliated Hospital of Jinzhou Medical University

Yuhong Li ( $\square$ yuhong_jiahui@163.com )

The First Affiliated Hospital of Jinzhou Medical University https://orcid.org/0000-0003-0056-8862

\section{Research Article}

Keywords: rheumatoid arthritis, ultrasound, ultrafast pulse wave velocity, carotid elasticity, inflammation, cardiovascular diseases

Posted Date: July 7th, 2021

DOl: https://doi.org/10.21203/rs.3.rs-673834/v1

License: (c) (i) This work is licensed under a Creative Commons Attribution 4.0 International License. Read Full License 


\section{Abstract}

\section{Purpose}

Ultrafast pulse wave velocity (UFPWV) is an innovative and practical technology that can assess the elastic function of blood vessels. This study aimed to explore the elastic function of the carotid artery using UFPWV and related influential factors in patients with rheumatoid arthritis (RA).

\section{Methods}

Overall, 120 patients with RA and 60 healthy controls were evaluated. Subjects underwent UFPWV and carotid artery intima-media thickness (cIMT) examination. The patients were divided into a mild activity group (group A) and moderate-to-severe activity group (group B). Differences in clinical indicators among the groups were determined, and the correlation between each indicator and PWV value was analyzed.

\section{Results}

The PWV values were higher in group $B$ than in group $A$ and were higher in group $A$ than in the control group $(P<0.05)$. The BS and ES $n$ the patient group were positively correlated with age, course of disease, systolic blood pressure (SBP), diastolic blood pressure, body mass index, total cholesterol, triglycerides, low-density lipoprotein cholesterol (LDL-C), cIMT, rheumatoid factor (RF), anti-cyclic citrullinated peptide (anti-CCP) antibody, erythrocyte sedimentation rate, C-reactive protein, and platelets and were negatively correlated with 25-hydroxyvitamin D3 $(25[\mathrm{OH}] \mathrm{D} 3)$ and hemoglobin $(\mathrm{P}<0.05)$. RF, anti-CCP antibody, age, course of disease, SBP, LDL-C, and platelets were risk factors for arteriosclerosis, whereas hemoglobin and 25(OH)D3 were protective factors $(P<0.05)$.

\section{Conclusion}

UFPWV allows for early and accurate detection of changes in arterial elasticity and enables early intervention to reduce the incidence of cardiovascular events in patients with RA.

\section{Introduction}

Rheumatoid arthritis (RA) is a chronic autoimmune disease that can involve several systems of the body [1]. The main pathological changes are synovitis and pannus formation with joint, cartilage, and bone destruction, which can eventually result in joint deformity and loss of function that can seriously affect a patient's quality of life [2]. The incidence of cerebrovascular and cardiovascular diseases (CVD) in patients with RA is significantly higher than that in the general population, and these diseases can shorten the life span of patients by approximately 5-10 years [3]. Atherosclerosis has become a recognized chronic inflammatory disease [4] and is a risk factor for CVD as it serves as an intermediate stage leading to cardiovascular events. Therefore, effective prevention and treatment of atherosclerosis in patients with RA can delay the development and progression of cardiovascular events. 
Changes in arterial elastic function occur before structural changes, such as thickening of arterial intimamedia and plaque formation. Ultrafast pulse wave velocity (UFPWV) technology adds a faster sampling frame rate on the basis of two-dimensional ultrasonography [5], making it an innovative imaging technology that can accurately evaluate arterial elastic function $[6,7]$. The purpose of this study was to evaluate the pulse wave velocity (PWV) of patients with RA using UFPWV technology and to explore related factors that may influence PWV. To the best of our knowledge, this is the first report in which UFPWV was used to study the elastic function of the carotid artery in patients with RA. UFPWV is an innovative and practical technology that provides a simple, reliable, and economical means for the early prevention and treatment of arteriosclerosis in patients with RA.

\section{Materials And Methods}

This was a prospective, observational, cross-sectional study

\section{Participants}

A total of 120 patients with RA who visited the Department of Rheumatology and Immunology of our hospital for the first time between March 2020 and March 2021 were included in this study. All patients were diagnosed based on the 2018 Chinese Guidelines for the Diagnosis and Treatment of Rheumatoid Arthritis.

The study population included 46 male and 74 female patients aged 18-70 years, with an average age of $55.42 \pm 10.15$ years. Patients were divided into a mild activity group (group A; Disease Activity Score 28 [DAS28] $\leq 3.2 ; 53$ cases) and a moderate-to-severe activity group (group B; DAS > 3.2; 67 cases) according to the DAS28 score of the RA disease activity scoring system recommended by the European League Against Rheumatism. In addition, 60 individuals who underwent a health checkup and whose age, sex, and body mass index (BMI) were matched with those in the study group were selected and included in the control group. The control group included 26 male and 34 female participants aged 1870 years, with an average age of $54.43 \pm 7.87$ years. Those with severe hypertension, severe CVDs, endocrine diseases, severe infections, other types of autoimmune diseases, severe liver and kidney dysfunction, hematological diseases, tumors, pregnancy, obesity, BMl of $\geq 35 \mathrm{~kg} / \mathrm{m}^{2}$, a history of longterm heavy cigarette smoking, long-term use of drugs that affect arterial elastic function, presence of carotid atherosclerotic plaque, carotid stenosis, and a tortuous carotid artery, as diagnosed by ultrasonography, were excluded.

\section{Main outcome variable}

All the above variables are obtained and examined by the Laboratory Department of the first affiliated Hospital of Jinzhou Medical University.

\section{Study factors}


The incidence of CVD in patients with RA is generally higher than that in the general population, and arteriosclerosis is the basis of CVD. The PWV of an artery is inversely proportional to its elasticity. Therefore, in this study, we used UFPWV technology to evaluate the carotid PWV in patients with RA and explore its related factors.

\section{Other variables}

Other variables evaluated were sex (male/female), age (years), systolic blood pressure (SBP) (mm $\mathrm{Hg})$, diastolic BP (DBP) (mm Hg), BMI $\left(\mathrm{kg} / \mathrm{m}^{2}\right)$, rheumatoid factor $(\mathrm{RF})(\mathrm{IU} / \mathrm{mL})$, anti-cyclic citrullinated peptide (anti-CCP) antibody ( $\mathrm{U} / \mathrm{mL}), \mathrm{C}$-reactive protein (CRP) $(\mathrm{mg} / \mathrm{L})$, erythrocyte sedimentation rate $(\mathrm{ESR})(\mathrm{mm} / \mathrm{h})$, hemoglobin $(\mathrm{Hb})(\mathrm{g} / \mathrm{L})$, platelets $(\mathrm{PLT})(\mathrm{x} 109 / \mathrm{L})$, total cholesterol

(TC) (mmol/L), triglycerides (TG) (mmol/L), low-density lipoprotein cholesterol (LDL-C) ( $\mathrm{mmol} / \mathrm{L})$, carotid artery intima-media thickness (cIMT) (mm), 25-hydroxyvitamin D3 (25[OH]D3) (ng/mL), PWV at the beginning of systole (PWV-BS) (m/s), and PWV at the end of systole (PWV-ES) (m/s).

\section{Procedures}

\section{Ultrasound examination}

PWV was performed at BS and ES. An Aixplorer ultrasonic diagnostic instrument with a built-in UFPWV (SuperSonic Imagine, Aix-en-Provence, France) was used. An SL10-2 probe was selected, frequency was set at $6-9 \mathrm{MHz}$, and carotid conditions were selected. Before the examination, the patient was instructed to rest quietly for $10 \mathrm{~min}$, to lay supine without a pillow, and to slightly lift the mandible to fully expose the neck. The maximum section of the longitudinal long axis at a distance of approximately $1-2 \mathrm{~cm}$ from the distal end of the bulbous part of the common carotid artery was selected to clearly display the intimamedia, the thickness of which was measured. The patient was instructed to hold their breath, then "PWV" was clicked, and the probe remained stable to facilitate the "acquiring and processing" function in order to process the images. The position of the sampling frame was adjusted so that the region of interest coincided with the carotid vessel wall. The "Select" button was clicked, and the PWV-BS and PWV-ES, as well as the standard deviation $(\Delta \pm)$, of the anterior wall of the carotid artery, were automatically measured. Data with $\Delta \pm \leq 20 \%$ were recorded as valid data. Each patient's carotid artery was measured at least three times on each side and the average value was recorded; all examinations were performed by two senior sonographers (Fig. 1).

\section{Laboratory examinations}

Laboratory test results were collected within 3 days after each patient's initial admission to the hospital. The tests included RF level, anti-CCP antibody titer, ESR, CRP level, Hb concentration, PLT, as well as $25(\mathrm{OH}) \mathrm{D} 3, \mathrm{TC}, \mathrm{TG}$, and LDL-C levels. After resting quietly for $30 \mathrm{~min}$, the participants' blood pressures were measured. Height and weight were measured to calculate BMI. The details of each patient's medical history and disease course of RA were collected. 
This study was approved by the Medical Ethics Committee of our hospital and informed consent was obtained from each subject (approval number: 202059).

\section{Statistical analysis}

SPSS 3.0 statistical software (IBM Corp., Armonk, NY, USA) was used for data analysis. Measurement data are expressed as $\overline{\mathrm{x}} \pm \mathrm{s}$. Enumeration data were compared using the $\mathrm{c}^{2}$ test. For normally distributed data, differences between the two groups were compared using independent samples t-test. Correlation analyses were performed using Pearson's correlation analysis. Influencing factors were analyzed using a multiple linear stepwise regression analysis. The inclusion and exclusion criteria were P-value of $<0.05$ and $>0.10$, respectively. P-value of $<0.05$ was considered to indicate a statistically significant difference. Intraclass correlation coefficients (ICCs) were adopted to evaluate the consistency of the tests.

\section{Results}

\section{Comparison of clinical indicators among patient and control groups}

The SBP; DBP; ESR; levels of RF, anti-CCP antibody, CRP, and PLT; and CIMT in the patient group were higher than those of participants in the control group $(\mathrm{P}<0.05)$. 25(OH)D3 and $\mathrm{Hb}$ levels were lower in the patient group than those in the control group $(P<0.05)$. There is no significant difference in terms of sex, age, BMI, TC, TG, or LDL-C between the two groups $(P>0.05)$, as shown in Table 1.

\section{Comparison between group A and group B}

The course of the disease was shorter, and SBP, DBP, ESR, and levels of RF, anti-CCP antibody, CRP, TC, TG, LDL-C, as well as PLT levels were lower in group $A$ than those in group $B(P<0.05)$. The levels of $25(\mathrm{OH}) \mathrm{D} 3$ and $\mathrm{Hb}$ were higher in group $A$ than those in group $B(P<0.05)$. However, there is no significant difference in terms of sex, BMI, or clMT between both groups $(P>0.05)$, as shown in Table 2 .

\section{Comparison of PWV-BS and PWV-ES among the groups}

There was no statistically significant difference in the PWV-BS or PWV-ES values of the left and right common carotid arteries among group $A$, group $B$, and the control group $(P>0.05)$. Therefore, the $P W V$ values were averaged. There were statistically significant differences in the PWV-BS and PWV-ES values among group $A$, group $B$, and the control group $(P<0.05)$. The values of $P W V-B S$ and $P W V-E S$ in group $B$ were greater than those in group $A(P<0.05)$. The values of PWV-BS and PWV-ES in group $A$ were greater than those in the control group $(P<0.05)$ (Tables 1 and 2$)$.

\section{Comparison of PWV values according to sex}

The patient group $(n=120)$ was divided into male $(n=46)$ and female $(n=74)$ groups. An independent samples t-test comparing the two groups showed that the PWV value was higher in the male group than 
that in the female group (BS: $6.63 \pm 0.83$ vs. $6.21 \pm 0.87 ; t=2.57 ; P<0.05 ; E S: 8.85 \pm 1.53$ vs. $7.86 \pm 1.30$; $t=3.83 ; P<0.05)$.

\section{Correlation analysis}

PWV-BS and PWV-ES were positively correlated with the course of disease, age, SBP, DBP, BMI, TC, TG, LDL-C, IMT, RF, anti-CCP antibody, ESR, CRP, and PLT count $(\mathrm{P}<0.05)$ and negatively correlated with $25(\mathrm{OH}) \mathrm{D}_{3}$ and $\mathrm{Hb}(\mathrm{P}<0.05)($ Table 3; Fig 2)

Analysis of influencing factors

In the stepwise regression analysis, PWV-BS and PWV-ES were taken as dependent variables, and age, course of disease, BMI, SBP, DBP, TC, TG, LDL-C, IMT, RF, anti-CCP antibody, ESR, CRP, PLT, 25(OH)D3 level, and $\mathrm{Hb}$ were taken as independent variables. The results of multiple linear regression analysis showed that the factors affecting PWV-BS included anti-CCP antibody, SBP, age, Hb, LDL-C, RF, course of disease, and 25(OH)D3. Factors affecting PWV-ES included anti-CCP antibody, age, SBP, Hb, PLT, LDL-C, and RF (Table 4).

\section{Results of consistency check}

ICCs were applied to evaluate the intra-examiner and inter-examiner reproducibility or consistency of examination results, and an ICC of $>0.75$ was considered to indicate good consistency. The results of PWV-BS and PWV-ES measured independently by two doctors showed good intra-examiner and interexaminer consistency $(\mathrm{ICC}>0.75)$.

\section{Discussion}

RA is currently the most common autoimmune disease, and the risk of cardiovascular events in patients with RA has increased by $48 \%$ compared to that of the general population [5]. After excluding traditional cardiovascular risk factors, such as diabetes, hyperlipidemia, hypertension, and obesity, this study found that the indicators of PWV were still higher in patients with RA than in subjects in the control group, indicating that the probability of arteriosclerosis was still higher in patients with RA than in healthy individuals after excluding traditional factors. This is related to the patient's long-term, high-level, inflammatory accumulation and immune disorder reaction $[5,6]$. In a severe chronic inflammatory state, the molecular structure of lipoprotein becomes smaller and more compact. This change induces the production of LDL-C and promotes arteriosclerosis, which reduces nitric oxide production and promotes reactive oxygen species production, leading to vascular endothelium damage [7]. The initial process of arteriosclerosis pathogenesis is usually endothelial injury and dysfunction. This is characterized by decreased vasodilation function, increased adhesion of large numbers of inflammatory cells and platelets, and increased procoagulant activity. The results of this study showed that the CRP, ESR, PLT, and LDL-C levels were higher in patients with RA than in healthy people, and LDL-C was a risk factor for PWV-BS and PWV-ES. This result is consistent with the above-mentioned mechanism. Atherosclerosis in 
patients with RA is not only closely related to inflammatory factors but also involves platelets. There are a large number of platelets in synovial lesions of patients with RA in the active stage of the disease. This is because synovial cells produce large amounts of pro-inflammatory factors, such as interleukin (IL)-1, IL-2, and IL-6, and these factors promote the proliferation and maturation of macrophages and induce the production of platelets, expand and maintain the inflammatory response, and increase inflammatory damage to the body. Furthermore, thrombocytosis causes patients with RA to be in a hypercoagulable state, and this accelerates arteriosclerosis. This easily results in an increased risk of a series of cardiovascular events, such as rupture and bleeding of atherosclerotic plaques and formation of arterial thromboembolism.

Atherosclerosis in patients with RA is also closely associated with high levels of ESR, CRP, and other indicators. The main mechanism involves action on the adipose tissue, skeletal muscles, liver, vascular endothelium, and other tissues, which results in a series of complex changes, including oxidative stress, insulin resistance, lipid metabolism dysfunction, and endothelial dysfunction, ultimately leading to arteriosclerosis [8]. Even if patients with RA do not have traditional risk factors for arteriosclerosis, they experience endothelial damage and functional changes [5], which may be associated with specific antibodies, such as RF and anti-CCP antibodies. The results of this study revealed that RF and anti-CCP antibody levels were higher in patients with RA than in healthy people, and the levels of RF and anti-CCP antibodies were higher in the moderate-to-severe activity group than in the mild activity group. Anti-CCP antibodies and RF are risk factors for PWV-BS and ES. This result is consistent with those of previous studies. RF tends to indicate clinical inflammatory activities. The higher the RF titer in patients with RA, the more active their inflammatory response. RF is an antibody against denatured immunoglobulin (Ig)G antigen (produced by bacteria, viruses, and other pathogens in the body), and generally present as IgMRF. IgM-RF is regarded as the iconic autoantibody of clinical RA and it may interact with the fragment crystallizable fragments of Ig molecules, mainly $\lg M$, $\lg D$, IgE, IgA, and IgG5. Patients with RF-positive RA have a higher risk of cardiovascular events than patients with RF-negative RA. RF is closely associated with a vascular injury in patients with RA. RF can directly produce toxic effects on vascular endothelial cells, resulting in endothelial injury and dysfunction, which is considered to be a risk factor for CVD in patients with RA. A positive anti-CCP antibody is closely correlated with endothelial dysfunction, increased cIMT, and arteriosclerosis. The American College of Rheumatology suggests that combined examinations of RF and anti-CCP antibodies are the best method for diagnosing RA [9].

Hypertension, diabetes, hyperlipidemia, smoking, and obesity have been proven to be associated with the pathogenesis of central vascular events in the general population $[10,11]$. The results of this study revealed that SBP, DBP, BMI, TC, and TG were positively correlated with PWV, and SDP was an influencing factor on PWV, which further confirms the correlation between RA and arteriosclerosis.

Related research has found that vitamin D promotes immunoregulatory activity, and this can inhibit the proliferation and differentiation of $\mathrm{T}$ cells, as well as the production of inflammatory cytokines and induction of the differentiation of regulatory $T$ cells [12]. Vitamin $D$ deficiency can increase the risk of autoimmune diseases such as type I diabetes, RA, ankylosing spondylitis, systemic lupus erythematosus, 
autoimmune thyroid disease, multiple sclerosis, and inflammatory bowel disease [13-15]. The results of a meta-analysis suggested that vitamin $D$ supplementation reduces the positive rate of anti-doublestranded deoxyribonucleic acid in systemic lupus erythematosus and may reduce the recurrence of RA [16]. 25(OH)D3 directly binds to vitamin $D$ receptors to participate in the physiological and pathological regulation of the cardiovascular system, especially in the regulation of endothelial cells and immune cells, which can lead to arteriosclerosis [17]. The results of our study revealed that 25(OH)D3 was negatively correlated with the PWV value, and 25(OH)D3 was a protective factor for PWV-BS; this is consistent with the mechanism described above.

In this study, the $\mathrm{Hb}$ level was significantly lower in the RA group than that in the control group, and $\mathrm{Hb}$ concentration was a protective factor for PWV-BS and PWV-ES. The possible mechanism is that with the disease progression in patients with RA, macrophages and T cells produce and release large amounts of interferons and tumor destruction factors that aggravate joint injury and bone destruction, inhibit the compensation of bone marrow erythrocytes, block the production of erythropoietin, and ultimately result in anemia [18]. This study revealed a negative correlation between $\mathrm{Hb}$ level and PWV value, suggesting that anemia may lead to disease deterioration in patients with RA and could affect their prognosis. Timely correction of anemia may alleviate this condition and delay the development of arteriosclerosis. This study showed that the course of the disease was positively correlated with PWV value and was a risk factor for PWV-BS, suggesting that long-term persistent chronic inflammation was a risk factor for arteriosclerosis. Therefore, patients with RA should be identified and treated as early as possible, as this can effectively prevent and treat the development and progression of arteriosclerosis.

This study showed that the PWV values and cIMT values in both the mild activity group and moderate-tosevere activity group of patients with RA were higher than those of patients in the control group, and the PWV value of those in the moderate-to-severe activity group was significantly higher than that of those in the mild activity group; however, there was no significant difference in the cIMT values between the two groups $(P>0.05)$, suggesting that arterial elastic function had changed in the mild activity group and the moderate-to-severe activity group. However, as a traditional marker for arteriosclerosis evaluation using ultrasonography, cIMT can rarely identify the difference in arteriosclerosis among patients with RA with different degrees of illness in early stages. cIMT is an important ultrasonic marker of arteriosclerosis and is associated with the degree of carotid artery stiffness $[19,20]$. Therefore, detecting changes in arterial function before significant thickening of cIMT has occurred is essential for the prevention and treatment of the disease.

Changes in the arterial elastic function can be examined by different non-invasive modalities. UFPWV is an emerging technology for accurately measuring carotid PWV in recent years. Its most significant characteristics are non-invasiveness, real-time measurement, simple operation, and high accuracy [21]. Because of its good stability and reliability in measuring vascular elasticity, it is considered to be the gold standard for evaluating the degree of arteriosclerosis [22]. UFPWV detects the PWV value using ultrafast imaging technology, with a frame rate of up to 2000 frames/s, and can accurately display and record the movement process of the arterial wall within $2 \mathrm{~s}$ and acquires and records the micro-movement speed 
and direction of the arterial wall by using a tissue Doppler imaging algorithm. It can also automatically calculate the PWV of measured local blood vessels at the beginning and at the end of systole and can obtain the BS and ES values [23] so as to evaluate changes in arterial elasticity. A larger PWV indicates poorer compliance and represents poorer arterial elastic function [22]. In contrast, traditional PWV measurement technologies, such as carotid-femoral PWV and brachial-ankle PWV, have larger errors in the measured PWV value owing to the complex detection technology and the influence of vascular tortuosity, among other reasons. We assessed the consistency of the findings of this study and found that UFPWV had high reproducibility and good stability; hence, it has great value in clinical application. Scholars from other countries have previously suggested that synovitis and arteriosclerosis in RA have similar immune mechanisms. Under the combined action of these complex factors, cardiovascular events have become the most common cause of death in patients with RA. Because these risk factors in the inflammatory environment change the structure and function of the arterial wall, the assessment of carotid PWV using UFPWV technology can be applied as an independent predictor of future CVD [24-26].

This study has some limitations. The comparison of PWV values among patients with RA of different ages and sexes in this study showed that the PWV values in males were significantly higher than those in females $(P<0.05)$, and this might be associated with the differences in hormone levels between males and females at different life stages. In this study, we did not analyze the hormone levels in patients of different ages and sexes or their possible effects on PWV. However, we plan to broaden the scope of our research in subsequent studies.

In conclusion, patients with RA should receive calcium supplements, their Hb levels should also be assessed regularly, and anemia should be corrected promptly. This cannot only avoid severe osteoporosis and bone destruction but will also protect their vascular endothelium and delay arteriosclerosis.

Traditional risk factors such as hypertension and hyperlipidemia; inflammatory indexes such as ESR, CRP, PLT, and LDL-C; and specific antibody RF (anti-CCP antibody) not only affect the severity of the disease but also cause irreversible damage to blood vessels and increase the risk of cardiovascular events in patients with RA. Therefore, the clinical treatment of patients with RA should involve paying close attention to these related indicators. With disease progression in patients with RA, PWV-BS and PWV-ES values also gradually increase, and arterial elasticity gradually decreases. UFPWV can provide a powerful reference base for the prevention and treatment of early-stage arteriosclerosis in patients with RA and thus should be widely promoted and applied in clinical practice.

\section{Declarations}

Funding: Liaoning Province's Plan for invigorating Liao talents (XLYC1802049)

Conflict of interestDThe authors declare that have no conflict of interest.

Availability of data and material: appliable.

Code availability: Not appliable. 
Ethical standards: The study was approved by the local Ethical Committee,and written informed consent was obtained from each participant.(Ethics approval number:202059)

\section{Ethics approval:}

This study was approved by the Medical Ethics Committee of the First Affiliated Hospital of Jinzhou Medical University. Informed consent was obtained from each subject.

Consent to participate: Not appliable.

\section{Acknowledgments}

We would like to thank Editage (www.editage.cn) for English language editing.

Consent for publication: Not appliable.

\section{References}

[1] Roth GA, Johnson C, Abajobir A et al (2015) Global, regional, and national burden of cardiovascular diseases for 10 causes, 1990 to 2015. J Am Coll Cardiol 70:1-25. https://doi.org/10.1016/j.jacc.2017.04.052

[2] Lillegraven S, van der Heijde D, Uhlig T et al (2012) What is the clinical relevance of erosions and joint space narrowing in RA? Nat Rev Rheumatol 8:117-120. https://doi.org/10.1038/nrrheum.2011.202

[3] Liu Y, Kaplan MJ (2018) Cardiovascular disease in systemic lupus erythematosus: an update. Curr Opin Rheumatol 30:441-448. https://doi.org/10.1097/BOR.0000000000000528

[4] Sébastien S, Chee A, Garcia D et al (2015) 2-D arterial wall motion imaging using ultrafast ultrasound and transverse oscillations. IEEE Trans Ultrason Ferroelectr Freq Control 62: 10471058. https://doi.org/10.1109/TUFFC.2014.006910

[5] Romana SF, Arbi P, Francesco C et al (2017) Association between antibodies to carbamylated proteins and subclinical atherosclerosis in rheumatoid arthritis patients. BMC Musculoskelet Disord 18:214. https://doi.org/10.1186/s12891-017-1563-8

[6] Karpouzas GA, Ormseth SR, Hernandez E et al (2020) Impact of cumulative inflammation, cardiac risk factors, and medication exposure on coronary atherosclerosis progression in rheumatoid arthritis. Arthritis Rheumatol 72:400-408. https://doi.org/10.1002/art.41122

[7] Zhang J, Chen L, Delzell E et al (2014) The association between inflammatory markers, serum lipids and the risk of cardiovascular events in patients with rheumatoid arthritis. Ann Rheum Dis 73:13011308. https://doi.org/10.1136/annrheumdis-2013-204715 
[8] Zamora C, Cantó E, Nieto JC et al (2017) Binding of platelets to lymphocytes: a potential antiinflammatory therapy in rheumatoid arthritis. J Immunol 198:3099-

3108. https://doi.org/10.4049/jimmunol.1601708

[9] El-Barbary AM, Kassem EM, El-Sergany MAS et al (2011) Association of anti-modified citrullinated vimentin with subclinical atherosclerosis in early rheumatoid arthritis compared with anti-cyclic citrullinated peptide. J Rheumatol 38:828-834. https://doi.org/10.3899/jrheum.101143

[10] Baghdadi LR, Woodman RJ, Shanahan EM et al (2015) The impact of traditional cardiovascular risk factors on cardiovascular outcomes in patients with rheumatoid arthritis: a systematic review and metaanalysis. PLoS One 10:e0117952. https://doi.org/10.1371/journal.pone.0117952

[11] Sparks JA, Chang SC, Nguyen US et al (2018) Weight change during the early rheumatoid arthritis period and risk of subsequent mortality in women with rheumatoid arthritis and matched comparators. Arthritis Rheumatol 70:18-29. https://doi.org/10.1002/art.40346

[12] Alhassan $\mathrm{MH}$, Saboor-Yaraghi AA, Vahedi $\mathrm{H}$ et al (2017) Immunomodulatory and immunosuppressive roles of 1a, 25(OH)2D3 in autoimmune diseases. Scand J Immunol 85:95103. https://doi.org/10.1111/sji.12512

[13] Jeffery LE, Raza K, Hewison M (2016) Vitamin D in rheumatoid arthritis-towards clinical application. Nat Rev Rheumatol 12:201-210. https://doi.org/10.1038/nrrheum.2015.140

[14] Guan SY, Cai HY, Wang P et al (2019) Association between circulating 25-hydroxyvitamin D and systemic lupus erythematosus: a systematic review and meta-analysis. Int J Rheum Dis 22:18031813. https://doi.org/10.1111/1756-185X.13676

[15] Bizzaro G, Shoenfeld Y (2015) Vitamin D and autoimmune thyroid diseases: facts and unresolved questions. Immunol Res 61:46-52. https://doi.org/10.1007/s12026-014-8579-z

[16] Franco AS, Freitas TQ, Bernardo WM et al (2017) Vitamin D supplementation and disease activity in patients with immune-mediated rheumatic diseases: a systematic review and meta-analysis. Medicine 96:e7024. https://doi.org/10.1097/MD.0000000000007024

[17] Kassi E, Adamopoulos C, Basdra EK, Papavassiliou AG (2013) Role of vitamin D in atherosclerosis. Circulation 128:2517-2531. https://doi.org/10.1161/CIRCULATIONAHA.113.002654

[18] Fukuda W, Omoto A, Oku S et al (2010) Contribution of rheumatoid arthritis disease activity and disability to rheumatoid cachexia. Mod Rheumatol 20:439-443. https://doi.org/10.1007/s10165-0100306-6

[19] Naqvi TZ, Lee MS (2014) Carotid intima-media thickness and plaque in cardiovascular risk assessment. JACC Cardiovasc Imaging 7:1025-1038. https://doi.org/10.1016/j.jcmg.2013.11.014 
[20] Den Ruijter HM, Peters SA, Anderson TJ et al (2012) Common carotid intimamedia thickness measurements in cardiovascular risk prediction: a meta-analysis. JAMA 308:796803. https://doi.org/10.1001/jama.2012.9630

[21] Goudot G, Mirault T, Khider L, et al (2019) Carotid stiffness assessment with ultrafast ultrasound imaging in case of bicuspid aortic valve. Front Physiol 10:1330. https://doi.org/10.3389/fphys.2019.01330

[22] Ben-Shlomo Y, Spears M, Boustred C et al (2014) Aortic pulse wave velocity improves cardiovascular event prediction: an individual participant meta-analysis of prospective observational data from 17,635 subjects. J Am Coll Cardiol 63:636-646. https://doi.org/10.1016/j.jacc.2013.09.063

[23] Tanter M, Fink M (2014) Ultrafast imaging in biomedical ultrasound. IEEE Trans Ultrason Ferroelectr Freq Control 61:102-119. https://doi.org/10.1109/TUFFC.2014.6689779

[24] Hannawi S, Hannawi H, Alokaily F et al (2018) Recent-onset of rheumatoid arthritis leads to increase in wall thickness of left anterior descending coronary artery. An evidence of subclinical coronary artery disease. Saudi Med J 39:1213-1217.

[25] Hannawi S, Hannawi H, Alokaily F et al (2017) Uric acid is independent cardiovascular risk factor, as manifested by increased carotid intima-media thickness in rheumatoid arthritis patients. Clin Rheumatol 36:1897-1902. https://doi.org/10.15537/smj.2018.12.23185

[26] Hannawi S, Hannawi H, Alokaily F et al (2020) Variables associated with subclinical atherosclerosis among rheumatoid arthritis patients of Gulf Cooperative Council countries. Saudi Med J 41:128137. https://doi.org/10.15537/smj.2020.2.24900

\section{Tables}


Table 1 Comparison of clinical indicators between the patient group and control group (mean \pm standard deviation)

\begin{tabular}{|c|c|c|c|c|}
\hline Variable & Patient group & Control group & $t / c^{2}$-value & P-value \\
\hline Sex (male/female) & $46 / 74$ & $26 / 34$ & 0.42 & 0.52 \\
\hline Age (years) & $55.42 \pm 10.15$ & $54.43 \pm 7.87$ & 0.72 & 0.48 \\
\hline SBP (mm Hg) & $131.45 \pm 14.16$ & $116.72 \pm 9.76$ & 8.16 & $<0.05$ \\
\hline DBP (mm Hg) & $79.67 \pm 8.78$ & $77.37 \pm 5.21$ & 2.2 & $<0.05$ \\
\hline $\mathrm{BMI}\left(\mathrm{kg} / \mathrm{m}^{2}\right)$ & $23.71 \pm 3.42$ & $23.42 \pm 3.45$ & 0.54 & 0.59 \\
\hline $\mathrm{RF}(\mathrm{IV} / \mathrm{mL})$ & $251.81 \pm 279.55$ & $0.95 \pm 1.64$ & 9.83 & $<0.05$ \\
\hline Anti-CCP antibody $(\mathrm{V} / \mathrm{mL})$ & $145.70 \pm 152.79$ & $1.32 \pm 0.71$ & 10.35 & $<0.05$ \\
\hline CRP (mg/L) & $46.61 \pm 43.01$ & $0.56 \pm 0.28$ & 11.73 & $<0.05$ \\
\hline $\operatorname{ESR}(\mathrm{mm} / \mathrm{h})$ & $44.04 \pm 27.80$ & $10.62 \pm 5.57$ & 12.67 & $<0.05$ \\
\hline $\mathrm{Hb}(\mathrm{g} / \mathrm{L})$ & $111.55 \pm 17.41$ & $134.52 \pm 13.53$ & -9.73 & $<0.05$ \\
\hline $\operatorname{PLT}\left(\times 10^{9} / \mathrm{L}\right)$ & $391.99 \pm 183.47$ & $243.82 \pm 58.72$ & 8.06 & $<0.05$ \\
\hline TC (mmol/L) & $4.28 \pm 1.01$ & $4.16 \pm 0.90$ & 0.79 & 0.43 \\
\hline TG (mmol/L) & $1.25 \pm 0.75$ & $1.19 \pm 0.75$ & 0.53 & 0.59 \\
\hline LDL-C (mmol/L) & $2.94 \pm 0.67$ & $2.88 \pm 0.65$ & 0.56 & 0.57 \\
\hline clMT (mm) & $0.59 \pm 0.11$ & $0.49 \pm 0.13$ & 5.26 & $<0.05$ \\
\hline 25(OH)D3 (ng/mL) & $11.85 \pm 5.36$ & $32.19 \pm 3.48$ & -30.63 & $<0.05$ \\
\hline PWV-BS (m/s) & $6.37 \pm 0.88$ & $4.00 \pm 0.33$ & 26.25 & $<0.05$ \\
\hline PWV-ES (m/s) & $8.24 \pm 1.47$ & $5.77 \pm 0.89$ & 13.97 & $<0.05$ \\
\hline
\end{tabular}

SBP, systolic blood pressure; DBP, diastolic blood pressure; BMI, body mass index; RF, rheumatoid factor; IV, intravenous; CCP, cyclic citrullinated peptide; CRP, C-reactive protein; ESR, erythrocyte sedimentation rate; Hb, hemoglobin; PLT, platelets; TC, total cholesterol; TG, triglycerides; LDL-C, low-density lipoprotein cholesterol; cIMT, carotid artery intima-media thickness; PWV-BS, pulse wave velocity at beginning of systole; PWV-ES, pulse wave velocity at end of systole; 25(OH)D3, 25-hydroxyvitamin D3 
Table 2 Comparison of indicators between the mild activity group (group A) and moderate-to-severe activity group (group B)

\begin{tabular}{|c|c|c|c|c|}
\hline Variable & Group A & Group B & $t / c^{2}$ & P-value \\
\hline Sex (male/female) & $21 / 32$ & $25 / 42$ & 0.07 & 0.77 \\
\hline Age (years) & $52.47 \pm 11.09$ & $57.75 \pm 8.74$ & -2.91 & $<0.05$ \\
\hline Course of disease (months) & $21.89 \pm 23.11$ & $45.96 \pm 51.98$ & -3.39 & $<0.05$ \\
\hline SBP $(\mathrm{mm} \mathrm{Hg})$ & $124.19 \pm 11.72$ & $137.19 \pm 13.31$ & -5.6 & $<0.05$ \\
\hline $\mathrm{DBP}(\mathrm{mm} \mathrm{Hg})$ & $76.23 \pm 8.28$ & $82.39 \pm 8.24$ & -4.06 & $<0.05$ \\
\hline BMI $\left(\mathrm{kg} / \mathrm{m}^{2}\right)$ & $23.11 \pm 3.51$ & $24.20 \pm 3.31$ & -1.74 & 0.09 \\
\hline $\mathrm{RF}(\mathrm{IV} / \mathrm{mL})$ & $123.73 \pm 129.21$ & $353.12 \pm 322.74$ & -5.31 & $<0.05$ \\
\hline Anti-CCP antibody (V/mL) & $73.09 \pm 90.71$ & $203.14 \pm 167.40$ & -5.43 & $<0.05$ \\
\hline CRP (mg/L) & $26.14 \pm 30.56$ & $62.80 \pm 44.70$ & -5.32 & $<0.05$ \\
\hline $\operatorname{ESR}(\mathrm{mm} / \mathrm{h})$ & $31.96 \pm 17.39$ & $53.60 \pm 30.74$ & -4.86 & $<0.05$ \\
\hline $\mathrm{Hb}(\mathrm{g} / \mathrm{L})$ & $118.12 \pm 16.28$ & $106.35 \pm 16.61$ & 3.89 & $<0.05$ \\
\hline PLT (x10²/L) & $308.28 \pm 129.52$ & $458.21 \pm 193.37$ & -5.07 & $<0.05$ \\
\hline $\mathrm{TC}(\mathrm{mmol} / \mathrm{L})$ & $4.02 \pm 0.85$ & $4.48 \pm 1.09$ & -2.58 & $<0.05$ \\
\hline TG (mmol/L) & $0.99 \pm 0.57$ & $1.47 \pm 0.82$ & -3.79 & $<0.05$ \\
\hline LDL-C (mmol/L) & $2.65 \pm 0.54$ & $3.16 \pm 0.67$ & -4.59 & $<0.05$ \\
\hline 25(OH)D3 (ng/mL) & $13.19 \pm 5.40$ & $10.79 \pm 5.11$ & 2.49 & $<0.05$ \\
\hline clMT (mm) & $0.06 \pm 0.01$ & $0.06 \pm 0.01$ & -1.71 & 0.09 \\
\hline PWV-BS (m/s) & $5.59 \pm 0.62$ & $7.00 \pm 0.43$ & -13.97 & $<0.05$ \\
\hline PWV-ES (m/s) & $7.08 \pm 0.97$ & $9.15 \pm 1.11$ & -10.73 & $<0.05$ \\
\hline
\end{tabular}

SBP, systolic blood pressure; DBP, diastolic blood pressure; BMI, body mass index; RF, rheumatoid factor; IV, intravenous; CCP, cyclic citrullinated peptide; CRP, C-reactive protein; ESR, erythrocyte sedimentation rate; $\mathrm{Hb}$, hemoglobin; PLT, platelets; TC, total cholesterol; TG, triglycerides; LDL-C, low-density lipoprotein cholesterol; cIMT, carotid artery intima-media thickness; PWV-BS, pulse wave velocity at beginning of systole; PWV-ES, pulse wave velocity at end of systole; $25(\mathrm{OH}) \mathrm{D} 3$, 25-hydroxyvitamin D3

Table 3 Correlation analysis of PWV-BS and PWV-ES with various clinical factors

Variable

Course of disease (months)

PWV-BS (m/s)
PWV-ES (m/s)

r-value

P-value

0.73 $<0.05$ 


\begin{tabular}{lllll} 
Age (years) & 0.62 & $<0.05$ & 0.73 & $<0.05$ \\
\hline SBP $(\mathrm{mm} \mathrm{Hg})$ & 0.45 & $<0.05$ & 0.48 & $<0.05$ \\
\hline $\mathrm{DBP}(\mathrm{mm} \mathrm{Hg})$ & 0.32 & $<0.05$ & 0.34 & $<0.05$ \\
\hline $\mathrm{BMI}\left(\mathrm{kg} / \mathrm{m}^{2}\right)$ & 0.52 & $<0.05$ & 0.58 & $<0.05$ \\
\hline $\mathrm{RF}(\mathrm{IV} / \mathrm{mL})$ & 0.52 & $<0.05$ & 0.58 & $<0.05$ \\
\hline Anti-CCP antibody $(\mathrm{V} / \mathrm{mL})$ & 0.67 & $<0.05$ & 0.8 & $<0.05$ \\
\hline $\mathrm{CRP}(\mathrm{mg} / \mathrm{L})$ & 0.38 & $<0.05$ & 0.43 & $<0.05$ \\
\hline $\mathrm{ESR}(\mathrm{mm} / \mathrm{h})$ & 0.38 & $<0.05$ & 0.43 & $<0.05$ \\
\hline $\mathrm{Hb}(\mathrm{g} / \mathrm{L})$ & -0.43 & $<0.05$ & -0.44 & $<0.05$ \\
\hline $\mathrm{PLT}\left(\mathrm{x} 10^{9} / \mathrm{L}\right)$ & 0.56 & $<0.05$ & 0.67 & $<0.05$ \\
\hline $\mathrm{TC}(\mathrm{mmol} / \mathrm{L})$ & 0.52 & $<0.05$ & 0.67 & $<0.05$ \\
\hline $\mathrm{TG}(\mathrm{mmol} / \mathrm{L})$ & 0.5 & $<0.05$ & 0.63 & $<0.05$ \\
\hline LDL-C (mmol/L) & 0.53 & $<0.05$ & 0.57 & $<0.05$ \\
\hline $\mathrm{CIMT}(\mathrm{mm})$ & 0.26 & $<0.05$ & 0.3 & $<0.05$ \\
\hline $25(\mathrm{OH}) \mathrm{D} 3(\mathrm{ng} / \mathrm{mL})$ & -0.41 & $<0.05$ & -0.57 & $<0.05$ \\
\hline
\end{tabular}

SBP, systolic blood pressure; DBP, diastolic blood pressure; BMI, body mass index; RF, rheumatoid factor; IV, intravenous; CCP, cyclic citrullinated peptide; CRP, C-reactive protein; ESR, erythrocyte sedimentation rate; Hb, hemoglobin; PLT, platelets; TC, total cholesterol; TG, triglycerides; LDL-C, low-density lipoprotein cholesterol; cIMT, carotid artery intima-media thickness; PWV-BS, pulse wave velocity at beginning of systole; PWV-ES, pulse wave velocity at end of systole; 25(OH)D3, 25-hydroxyvitamin D3

SBP, systolic blood pressure; DBP, diastolic blood pressure; RF, rheumatoid factor; CCP, cyclic citrullinated peptide; $\mathrm{Hb}$, hemoglobin; PLT, platelets; LDL-C, low-density lipoprotein cholesterol; PWV-BS, pulse wave velocity at beginning of systole; PWV-ES, pulse wave velocity at end of systole; $25(\mathrm{OH}) \mathrm{D} 3,25-$ hydroxyvitamin D3

\section{Figures}


Table 4 Multiple stepwise regression analysis of influencing factors on PWV-BS and PWV-ES

\begin{tabular}{|c|c|c|c|c|c|c|}
\hline \multirow[t]{2}{*}{$\begin{array}{l}\text { Independent } \\
\text { variable }\end{array}$} & \multirow[t]{2}{*}{$\begin{array}{l}\text { Independent } \\
\text { variable }\end{array}$} & \multicolumn{2}{|c|}{$\begin{array}{l}\text { Unstandardized } \\
\text { coefficient }\end{array}$} & \multirow[t]{2}{*}{$\begin{array}{l}\text { Standardized } \\
\text { coefficient } \beta\end{array}$} & \multirow[t]{2}{*}{$\begin{array}{l}\mathrm{t}- \\
\text { value }\end{array}$} & \multirow[t]{2}{*}{$\begin{array}{l}\mathrm{P} \text { - } \\
\text { value }\end{array}$} \\
\hline & & $\beta$ & $\begin{array}{l}\text { Standard } \\
\text { error }\end{array}$ & & & \\
\hline \multirow[t]{8}{*}{ PWV-BS } & $\begin{array}{l}\text { Anti-CCP } \\
\text { antibody }\end{array}$ & 0.002 & 0.001 & 0.27 & 2.98 & 0.004 \\
\hline & SBP & 0.017 & 0.004 & 0.27 & 4.45 & 0 \\
\hline & Age & 0.033 & 0.008 & 0.38 & 4.21 & 0 \\
\hline & $\mathrm{Hb}$ & -0.01 & 0.003 & -0.21 & -3.58 & 0.001 \\
\hline & LDL-C & 0.261 & 0.087 & 0.2 & 3 & 0.003 \\
\hline & RF & 0.001 & 0 & 0.2 & 2.97 & 0.004 \\
\hline & $\begin{array}{l}\text { Course of } \\
\text { disease }\end{array}$ & 0.026 & 0.012 & 0.16 & 2.09 & 0.027 \\
\hline & 25(OH)D3 & -0.004 & 0.002 & -0.19 & -2.23 & 0.039 \\
\hline \multirow[t]{7}{*}{ PWV-ES } & $\begin{array}{l}\text { Anti-CCP } \\
\text { antibody }\end{array}$ & 0.002 & 0.001 & 0.26 & 3.9 & 0 \\
\hline & Age & 0.043 & 0.008 & 0.3 & 5.2 & 0 \\
\hline & SBP & 0.017 & 0.005 & 0.17 & 3.46 & 0.001 \\
\hline & $\mathrm{Hb}$ & -0.015 & 0.004 & -0.18 & -4.22 & 0 \\
\hline & PLT & 0.001 & 0 & 0.16 & 2.59 & 0.011 \\
\hline & LDL-C & 0.289 & 0.108 & 0.13 & 2.67 & 0.009 \\
\hline & RF & 0.001 & 0 & 0.13 & 2.54 & 0.013 \\
\hline
\end{tabular}



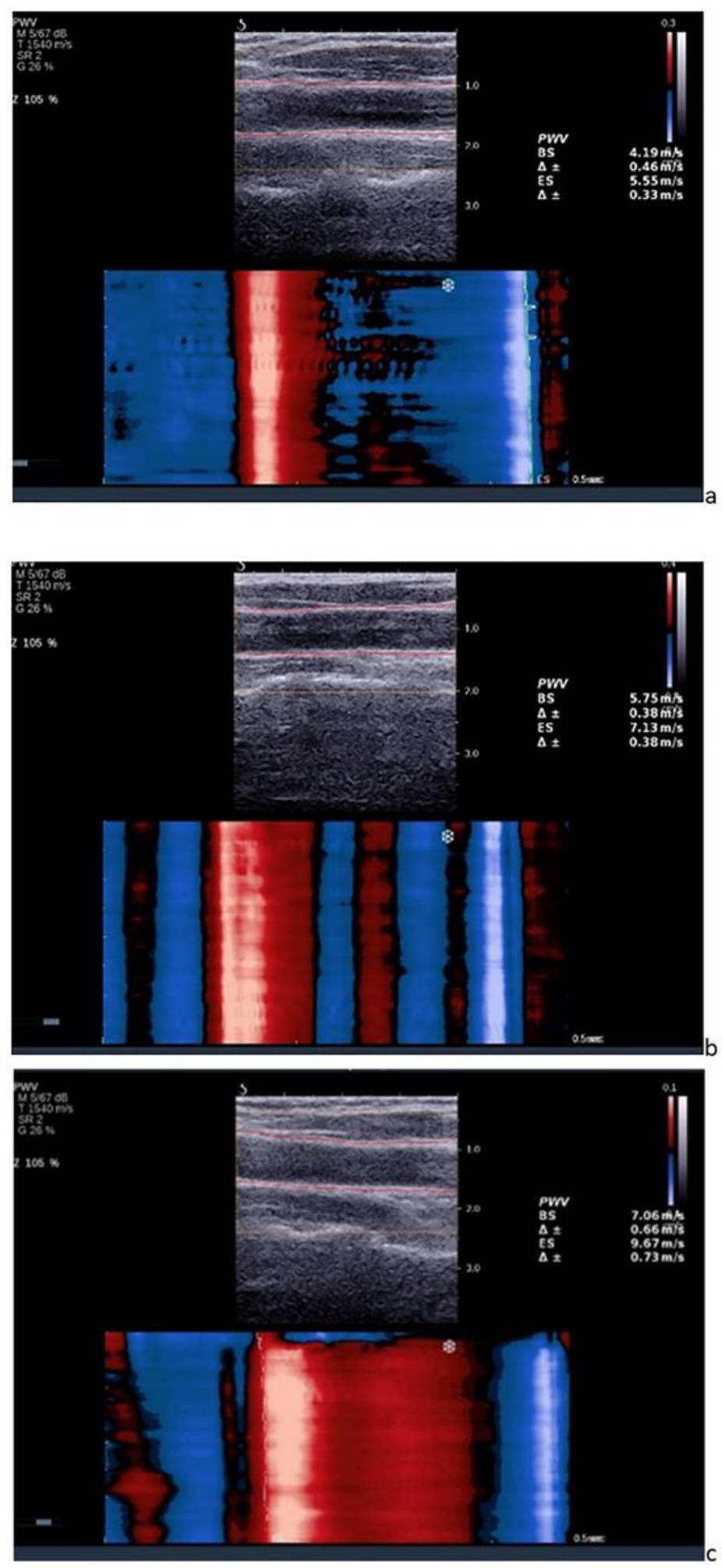

\section{Figure 1}

a. A healthy 45-year-old male undergoing a health checkup, PWV-BS: $4.19 \mathrm{~m} / \mathrm{s}$; PWV-ES: $5.55 \mathrm{~m} / \mathrm{s} ; \mathrm{b}$. A 56-year-old female in the mild activity group; PWV-BS: $5.75 \mathrm{~m} / \mathrm{s}$; PWV-ES: $7.13 \mathrm{~m} / \mathrm{s}$; c. A 51-year-old male in the moderate-to-severe activity group; PWV-BS: $7.06 \mathrm{~m} / \mathrm{s}$; PWV-ES: $9.67 \mathrm{~m} / \mathrm{s}$ Abbreviations: PWV-BS, pulse wave velocity at beginning of systole; PWV-ES, pulse wave velocity at end of systole 

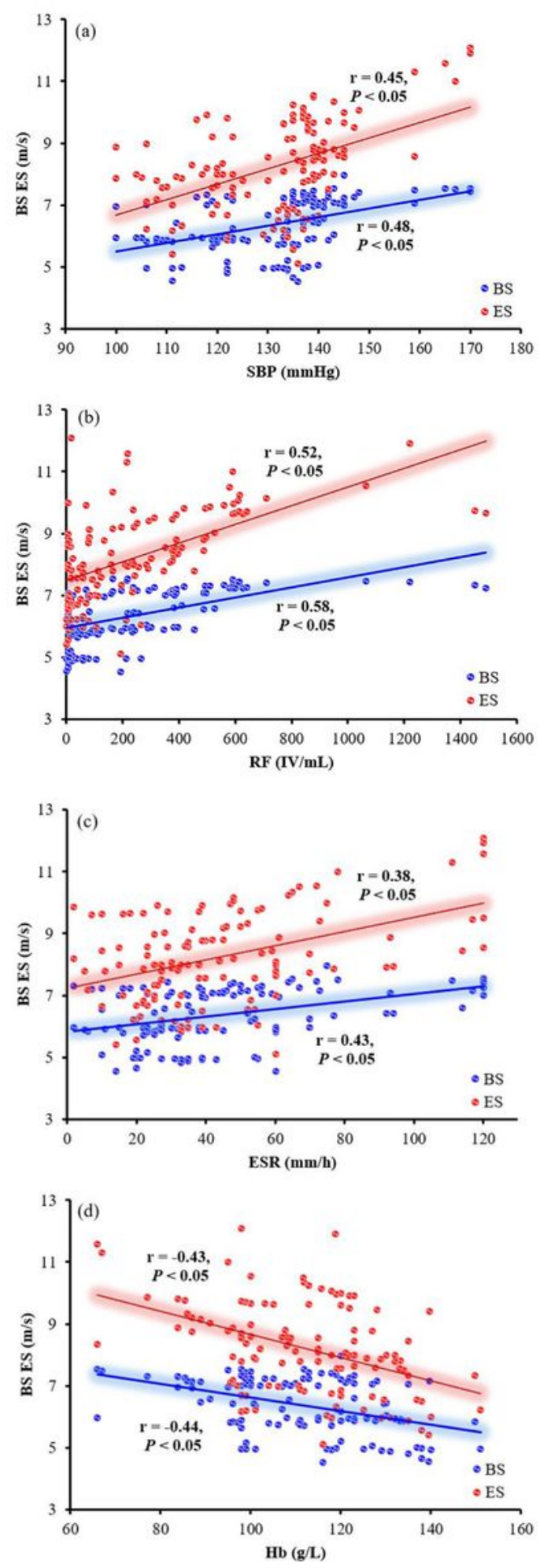

\section{Figure 2}

a. SBP is positively correlated with PWV-BS and PWV-ES in the patient group $(r=0.45$ and 0.48 , respectively; $P<0.05$ ); $b$. RF is positively correlated with PWV-BS and PWV-ES in the patient group ( $r=$ 0.52 and 0.58 , respectively; $\mathrm{P}<0.05$ ); $\mathrm{C}$. The ESR is positively correlated with PWV-BS and PWV-ES in the patient group ( $r=0.38$ and 0.43 , respectively; $P<0.05) ; d$. $\mathrm{Hb}$ is negatively correlated with PWV-BS and PWV-ES in the patient group $(r=-0.43$ and -0.44 , respectively; $P<0.05)$ Abbreviations: SBP, systolic 
blood pressure; RA, rheumatoid arthritis; RF, rheumatoid factor; ESR, erythrocyte sedimentation rate; $H B$, hemoglobin; PWV-BS, pulse wave velocity at beginning of systole; PWV-ES, pulse wave velocity at end of systole 